\title{
Some Estimation Methods for Right Censored Data of Type II Related to Gumbel Distribution
}

\author{
'Fadi A. Shaayo and Akram M. Al-Abood \\ Department of Mathematics and Computer Applications, College of Sciences, \\ Al-Nahrain University, Baghdad-Iraq. \\ 'E-mail: master_fadi@yahoo.com.
}

\begin{abstract}
In this paper, right censored data of type II related to the Gumbel distribution (maximum extreme value distribution) of two parameters is considered. Estimation of the distribution parameters are presented by two methods: maximum likelihood method and modified moment method. Moments properties of the estimators such as bias, variance, skewness and kurtosis are compared between two methods. We show that the estimators are unbiased, and its variances converge to zero, which the estimators are consistent in modified moment method. Consistency of the maximum likelihood and modified moment estimators are tabulated by using mean square error. Confidence interval estimation for the distribution parameters based on the maximum likelihood method and modified moment method are given by Monte Carlo simulation.
\end{abstract}

Keywords: Gumbel Distribution, Censored Data, Maximum Likelihood Method, Modified Moment Method, Monte Carlo Simulation.

\section{Introduction}

Recently there has been a great deal of interest in the use of various types of parametric models for the analysis of what is variously referred to as lifetime, survival time or failure time data that have as end point the time until the failure occurs. The major areas of application of such models appear in medical studies of chronic diseases, in industrial life testing, flood frequency analysis, reliability analysis in engineering, financial risk management, etc. $\left[{ }^{\vee}\right]\left[{ }^{9}\right]$

In survival data investigation, it is quite common to find some units have not failed when the observation is terminated. There failure times are therefore unknown but it is known they exceed their survival times measured at the end of investigation. Such failure times are said to be right censored. This censoring mechanism may occur due to the need for early termination of the investigation or removal of units from use before failure, or failure of units may occur because of causes unrelated to the application of the operating conditions, etc., and where records of survival times cannot subsequently be obtained. $[\mathrm{V}]\left[{ }^{\mathrm{Q}}\right]$

Censored data are said to have type I censoring, if censored observation occur only at specified values of the dependent variables, for example, in life testing when all units are put on test at the same time, data are collected and analyzed at a specific point in time. In this type of censoring, the censoring values are fixed and the number of censored observation is random. [ $][Y][1 \cdot]$

Censored data are said to have type II censoring, if the number of censored observation is specified and their censored values are random. A hybrid censoring scheme is a mixture of Type-I and Type-II censoring schemes. Progressive Type-II hybrid censoring is a mixture of progressive Type-II and hybrid censoring schemes. [ 1$]\left[{ }^{r}\right][1 \cdot]$

Some useful references to life data, model representation and associated statistical analyses are given by, Balakrishnan et al. $(Y \cdots \varepsilon)[Y]$, discussed in classical framework, the point and interval estimation for parameters of the extreme value distribution based on progressively Type-II censored data. Thompson et. al. $(r \cdot 11)\left[{ }^{9}\right]$, introduced a distributional hypothesis test for left censored Gumbel observations based on the probability plot correlation coefficient. Salinas et. al. $(r \cdot, r)[\urcorner]$, considered goodness of fit tests for the Gumbel distribution with type II right censored data. El Sherpieny et. al. $(r \cdot 1 r)[r]$, considered the estimation for the three unknown parameters of the generalized extreme value distribution under progressive 
type-II censored, and obtained the corresponding asymptotic variance covariance matrix for the parameters and also asymptotic confidence intervals for the parameters. Fard and Holmquist $(r \cdot 1 r)[r]$, investigated several procedures for goodness-of-fit of the extreme value distribution and the procedures make use of recent available accurate approximations of the means and variances of order statistics from the standardized extreme value distribution, and are either modifications in estimation techniques of earlier proposed test statistics or are newly introduced test statistics based on the regression of the order statistics on their means. Sheng $(r+1 r)[V]$ has been applied order-statistics-based inferences on lifetime analysis and financial risk measurement. He gives three problems, one of the problems which are raised from fitting the Weibull distribution to progressively censored and accelerated life-test data, and gives a new order-statistics-based inference is proposed for both parameter and confidence interval estimation. $\mathrm{Wu}$ et. al. $\left(r^{\prime} \mid r\right)[1 \cdot]$, proposed the weighted moments estimators (WMEs) of the location and scale parameters for the extreme value distribution based on the multiply type II censored sample, and its compared by using simulation mean squared errors (MSEs) of best linear unbiased estimator (BLUE) and exact MSEs of WMEs to study the behavior of different estimation methods, and that the results show the best estimator among the WMEs and BLUE under different combinations of censoring schemes.

In this paper, we use maximum likelihood method and modified moment method to estimate the parameters of Gumbel distribution (maximum extreme value distribution) of right censored data of type II. We show that the estimators are unbiased and its variances converge to zero in modified moment method, which the estimators are consistent. Finally, the confidence interval estimation for the parameters distribution based on maximum likelihood method and modified moment method were found.

\section{Right Censored Data Related to Gumbel Distribution of Type II}

Let $X, X_{r}, \ldots, X_{n}$ represent the time to failures of the $n$ component and let $Y_{1}, Y_{r}, \ldots$, $Y_{n}$ represent their arrangement in ascending order of magnitude, then from order statistic theory, the joint p.d.f. of $r$ censored data of $Y_{1}$, $Y_{r}, \ldots, Y_{r},(r<n)($ Hogg and Graig $(19 \vee \wedge))$ [?] is:

$\mathrm{g}\left(\mathrm{y}, \mathrm{y}^{\mathrm{r}}, \ldots, \mathrm{y}_{\mathrm{r}}\right)=\frac{\mathrm{n} !}{(\mathrm{n}-\mathrm{r}) !} \prod_{\mathrm{i}=1}^{\mathrm{r}} \mathrm{f}\left(\mathrm{y}_{\mathrm{i}}\right)\left[1-\mathrm{F}\left(\mathrm{y}_{\mathrm{r}}\right)\right]^{\mathrm{n}-\mathrm{r}}$,

$-\infty<\mathrm{y}_{1}<\mathrm{y}_{\mathrm{r}}<\ldots<\mathrm{y}_{\mathrm{r}}<\infty$

Consider $\mathrm{X}$ has p.d.f. from Gumbel distribution is

$\mathrm{f}(\mathrm{x})=\frac{1}{\beta} e^{-\left(\frac{x-\alpha}{\beta}\right)} e^{-e^{-\left(\frac{x-\alpha}{\beta}\right)}}$

$-\infty<\alpha<\infty, \beta>\cdot,-\infty<x<\infty$

where $\alpha$ is scale parameter and $\beta$ is shape parameter

and $\mathrm{X}$ has c.d.f. is

$\mathrm{F}(\mathrm{x})=\left\{\begin{array}{cl}\cdot, & x \rightarrow-\infty \\ e^{-e^{-\left(\frac{x-\alpha}{\beta}\right)},},-\infty<x<\infty \\ , x \rightarrow \infty\end{array}\right.$

Now let $\mathrm{Y}_{1}<\mathrm{Y}_{r}<\ldots<\mathrm{Y}_{\mathrm{r}}$ is the order statistic of a r.v. of size $r$ with p.d.f. and c.d.f. given from eqs. $(r)$ and $(r)$. Then using eq. ( () , we have

$$
\begin{aligned}
& \mathrm{L}=\mathrm{h}\left(\mathrm{y},, \mathrm{y}_{r}, \ldots, \mathrm{y}_{\mathrm{r}}\right) \\
&=\frac{\mathrm{n} !}{(\mathrm{n}-\mathrm{r}) !} \prod_{\mathrm{i}=1}^{\mathrm{r}}\left[\frac{1}{\beta} e^{-\left(\frac{\mathrm{y}_{\mathrm{i}}-\alpha}{\beta}\right)} e^{\left.-e^{-\left(\frac{\mathrm{y}_{\mathrm{i}}-\alpha}{\beta}\right)}\right]}\right. \\
& {\left[1-e^{\left.-e^{-\left(\frac{y_{r}-\alpha}{\beta}\right)}\right]^{\mathrm{n}-\mathrm{r}}}\right.} \\
&=\frac{\mathrm{n} !}{(\mathrm{n}-\mathrm{r}) !} \frac{1}{\beta^{r}} e^{-\sum_{i=1}^{r}\left(\frac{\mathrm{y}_{\mathrm{i}}-\alpha}{\beta}\right)} \\
& e^{-\sum_{i=1}^{r} e^{-\left(\frac{\mathrm{y}_{\mathrm{i}}-\alpha}{\beta}\right)}}\left(1-e^{-e^{-\left(\frac{y_{r}-\alpha}{\beta}\right)}}\right)^{n-r}
\end{aligned}
$$

Estimation of parameters for Gumbel distribution by Maximum Likelihood Method

We shall use maximum likelihood method for estimating the distribution parameters of $\alpha$ and $\beta$, where the logarithmic likelihood equation given by $(\varepsilon)$ is

$$
\begin{aligned}
& \ln (\mathrm{L})=\ln \left(\frac{n !}{(n-r) !}\right)-r \ln \beta-\sum_{i=1}^{r}\left(\frac{\mathrm{y}_{\mathrm{i}}-\alpha}{\beta}\right)- \\
& \sum_{i=1}^{r} e^{-\left(\frac{\mathrm{y}_{\mathrm{i}}-\alpha}{\beta}\right)} \\
& +(\mathrm{n}-\mathrm{r}) \ln \left(1-e^{-e^{-\left(\frac{y_{r}-\alpha}{\beta}\right)}}\right)
\end{aligned}
$$




\section{Journal of Al-Nahrain University}

\section{Science}

$\mathrm{f}_{1}=\frac{\partial \ln (\mathrm{L})}{\partial \alpha}=\frac{r}{\beta}-\frac{1}{\beta} \sum_{i=1}^{r} e^{-\left(\frac{\mathrm{y}_{\mathrm{i}}-\alpha}{\beta}\right)}$

$+\frac{(\mathrm{n}-\mathrm{r}) e^{-\left(\frac{y_{r}-\alpha}{\beta}\right)} e^{-e^{-\left(\frac{y_{r}-\alpha}{\beta}\right)}}}{\beta\left(1-e^{\left.-e^{-\left(\frac{y_{r}-\alpha}{\beta}\right)}\right)}\right.}$

$\mathrm{f}_{Y}=\frac{\partial \ln (\mathrm{L})}{\partial \beta}=\frac{-r}{\beta}+\frac{1}{\beta} \sum_{i=1}^{r}\left(\frac{\mathrm{y}_{\mathrm{i}}-\alpha}{\beta}\right)-$ $\frac{1}{\beta} \sum_{i=1}^{r}\left(\frac{\mathrm{y}_{\mathrm{i}}-\alpha}{\beta}\right) e^{-\left(\frac{\mathrm{y}_{\mathrm{i}}-\alpha}{\beta}\right)}$

$+\frac{\left(\frac{y_{r}-\alpha}{\beta}\right)(\mathrm{n}-\mathrm{r}) e^{-\left(\frac{y_{r}-\alpha}{\beta}\right)} e^{-e^{-\left(\frac{y_{r}-\alpha}{\beta}\right)}}}{\beta\left(1-e^{\left.-e^{-\left(\frac{y_{r}-\alpha}{\beta}\right)}\right)}\right.}$

Setting $\frac{\partial \ln (\mathrm{L})}{\partial \alpha}=\frac{\partial \ln (\mathrm{L})}{\partial \beta}=$, at $\alpha=\hat{\alpha}, \beta=\widehat{\beta}$, we have

$\frac{r}{\hat{\beta}}-\frac{1}{\hat{\beta}} \sum_{i=1}^{r} e^{-\left(\frac{\mathrm{y}_{\mathrm{i}}-\hat{\alpha}}{\hat{\beta}}\right)}+\frac{(\mathrm{n}-\mathrm{r}) e^{-\left(\frac{y_{r}-\hat{\alpha}}{\hat{\beta}}\right)} e^{-e^{-\left(\frac{y_{r}-\widehat{\alpha}}{\hat{\beta}}\right)}}}{\hat{\beta}\left(1-e^{\left.-e^{-\left(\frac{y_{r}-\hat{\alpha}}{\hat{\beta}}\right)}\right)}\right.}=\cdot$

$\frac{-r}{\hat{\beta}}+\frac{1}{\hat{\beta}} \sum_{i=1}^{r}\left(\frac{\mathrm{y}_{\mathrm{i}}-\widehat{\alpha}}{\hat{\beta}}\right)-\frac{1}{\hat{\beta}} \sum_{i=1}^{r}\left(\frac{\mathrm{y}_{\mathrm{i}}-\hat{\alpha}}{\hat{\beta}}\right) e^{-\left(\frac{\mathrm{y}_{\mathrm{i}}-\hat{\alpha}}{\hat{\beta}}\right)}+$ $\left(\frac{y_{r}-\hat{\alpha}}{\hat{\beta}}\right) \frac{(\mathrm{n}-\mathrm{r}) e^{-\left(\frac{y_{r}-\widehat{\alpha}}{\hat{\beta}}\right)} e^{-e^{-\left(\frac{y_{r}-\hat{\alpha}}{\hat{\beta}}\right)}}}{\hat{\beta}\left(1-e^{\left.-e^{-\left(\frac{y_{r}-\hat{\alpha}}{\hat{\beta}}\right)}\right)}\right.}=$

From eq.s $(\vee)$ and $(\wedge)$, which are non linear equations that there are no analytically solution can be made for $\hat{\alpha}$ and $\hat{\beta}$. So approximation to $\hat{\alpha}$ and $\hat{\beta}$ could be obtained by using Newton-Raphson. We could be obtained on the second and mixed partial derivatives of eq.s $\left({ }^{0}\right)$ and $(7)$ with respect to $\alpha$ and $\beta$ by the observe fisher information matrix for maximum likelihood estimation (El Sherpieny et. al. $(r \cdot \mid r))[r]$, which is:

$$
\begin{aligned}
I_{(\alpha, \beta)=(\hat{\alpha}, \widehat{\beta})} & =\left[\begin{array}{cc}
-\frac{\partial^{r} \ln (\mathrm{L})}{\partial \hat{\alpha}^{r}} & -\frac{\partial^{r} \ln (\mathrm{L})}{\partial \hat{\alpha} \partial \hat{\beta}} \\
-\frac{\partial^{r} \ln (\mathrm{L})}{\partial \hat{\beta} \partial \hat{\alpha}} & -\frac{\partial^{r} \ln (\mathrm{L})}{\partial \hat{\beta}^{r}}
\end{array}\right] \\
& =-\left[\begin{array}{ll}
a & b \\
b & c
\end{array}\right] \\
& =-\left[\begin{array}{cc}
\operatorname{var}(\hat{\alpha}) & \operatorname{cov}(\hat{\alpha}, \hat{\beta}) \\
\operatorname{cov}(\hat{\alpha}, \widehat{\beta}) & \operatorname{var}(\hat{\beta})
\end{array}\right]
\end{aligned}
$$

where

$\mathrm{a}=\frac{\partial^{\Upsilon} \ln (\mathrm{L})}{\partial \hat{\alpha}^{\Upsilon}}=\frac{-1}{\hat{\beta}^{\Upsilon}} \sum_{i=1}^{r} e^{-\left(\frac{y_{i}-\hat{\alpha}}{\hat{\beta}}\right)}+$

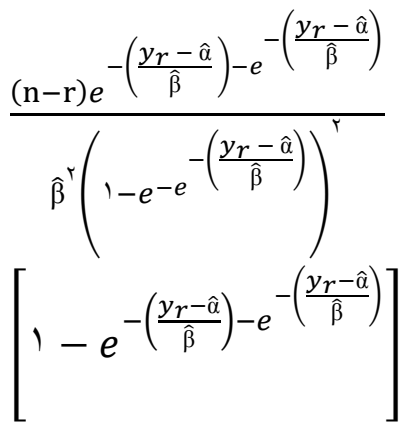

$\mathrm{b}=\frac{\partial^{\Upsilon} \ln (\mathrm{L})}{\partial \hat{\alpha} \partial \hat{\beta}}=\frac{\partial^{\Upsilon} \ln (\mathrm{L})}{\partial \hat{\beta} \partial \hat{\alpha}}=\frac{-r}{\hat{\beta}^{\Upsilon}}+\frac{1}{\hat{\beta}^{\Upsilon}} \sum_{i=1}^{r} e^{-\left(\frac{y_{i}-\hat{\alpha}}{\hat{\beta}}\right)}-$

$\frac{1}{\hat{\beta}} \sum_{i=1}^{r}\left(\frac{y_{i}-\hat{\alpha}}{\hat{\beta}}\right) e^{-\left(\frac{y_{i}-\hat{\alpha}}{\hat{\beta}}\right)}+$

$\frac{(\mathrm{n}-\mathrm{r}) e^{-\left(\frac{y_{r}-\hat{\alpha}}{\hat{\beta}}\right)-e^{-\left(\frac{y_{r}-\hat{\alpha}}{\hat{\beta}}\right)}}}{\widehat{\beta}^{r}\left(1-e^{\left.-e^{-\left(\frac{y_{r}-\widehat{\alpha}}{\hat{\beta}}\right)}\right)^{r}}\right.}\left[\left(\frac{y_{r}-\widehat{\alpha}}{\hat{\beta}}-r\right)-\right.$

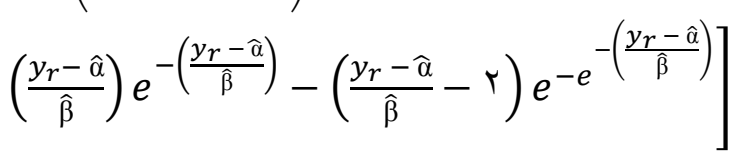

$\mathrm{c}=\frac{\partial^{\Upsilon} \ln (\mathrm{L})}{\partial \widehat{\beta}^{\Upsilon}}=\frac{r}{\hat{\beta}^{\Upsilon}}-\frac{r}{\hat{\beta}^{r}} \sum_{i=1}^{r}\left(\frac{y_{i}-\hat{\alpha}}{\hat{\beta}}\right)+$

$\left(r+\frac{1}{\hat{\beta}}\right) \sum_{i=\prime}^{r}\left(\frac{y_{i}-\widehat{\alpha}}{\hat{\beta}^{\top}}\right) e^{-\left(\frac{y_{i}-\hat{\alpha}}{\hat{\beta}}\right)}+$

$\frac{(\mathrm{n}-\mathrm{r})\left(\frac{y_{r}-\hat{\alpha}}{\hat{\beta}}\right)}{\hat{\beta}^{r}\left(1-e^{\left.-e^{-\left(\frac{y_{r}-\widehat{\alpha}}{\hat{\beta}}\right)}\right)}\right.}$

$\left[\left(\frac{y_{r}-\hat{\alpha}}{\hat{\beta}}-r\right) e^{-e^{-\left(\frac{y_{r}-\hat{\alpha}}{\hat{\beta}}\right)}} e^{-\left(\frac{y_{r}-\hat{\alpha}}{\hat{\beta}}\right)}-\right.$

$\left(\frac{y_{r}-\hat{\alpha}}{\hat{\beta}}\right) e^{-r\left(\frac{y_{r}-\hat{\alpha}}{\hat{\beta}}\right)} e^{-e^{-\left(\frac{y_{r}-\hat{\alpha}}{\hat{\beta}}\right)}}-\left(\frac{y_{r}-\hat{\alpha}}{\hat{\beta}}-\right.$

$\left.r) e^{-\left(\frac{y_{r}-\hat{\alpha}}{\hat{\beta}}\right)} e^{-r e^{-\left(\frac{y_{r}-\hat{\alpha}}{\hat{\beta}}\right)}}\right]$

to solve eq.s $(\vee)$ and $(\wedge)$ by using NewtonRaphson, let $\left(\hat{\alpha}^{(')}, \hat{\beta}^{(')}\right)$ be given initial approximation. If $\left(\hat{\alpha}^{(\mathrm{s})}, \widehat{\beta}^{(\mathrm{s})}\right)$ is the approximation solution of $(\hat{\alpha}, \widehat{\beta})$ at stage $(s)$, s $=1, r, r, \ldots .$. , then the approximation solution at stage $(\mathrm{s}+\mathrm{l})$

$\left[\begin{array}{c}\hat{\alpha}^{(s+1)} \\ \hat{\beta}^{(s+1)}\end{array}\right]=\left[\begin{array}{l}\hat{\alpha}^{(s)} \\ \hat{\beta}^{(s)}\end{array}\right]-\left[\begin{array}{ll}a & b \\ b & c\end{array}\right]^{-1}\left[\begin{array}{l}f_{Y}^{(s)} \\ f_{\Upsilon}(s)\end{array}\right]$ 
$=\left[\begin{array}{c}\hat{\alpha}^{(\mathrm{s})} \\ \hat{\beta}^{(\mathrm{s})}\end{array}\right]-\frac{1}{a c-b^{\Upsilon}}\left[\begin{array}{cc}c & -b \\ -b & a\end{array}\right]\left[\begin{array}{l}\mathrm{f}_{\mathrm{f}}{ }^{(\mathrm{s})} \\ \mathrm{f}_{\Upsilon}(\mathrm{s})\end{array}\right]$

where $a c-b^{r} \neq \cdot$.

The process is repeated until successive $\alpha$ and $\beta$ estimators agree to a specified tolerance on an element by element basis.

Estimation of parameters for Gumbel distribution by Modified Moment Method

We shall use modified moment method for estimating the distribution parameters of $\alpha$ and $\beta$. Let $Y$, is the first order statistic of $r$ censored data

$\mathrm{g}_{1}\left(\mathrm{y}_{\prime}\right)=\mathrm{n}\left[1-\mathrm{F}\left(y_{\curlywedge}\right)\right]^{\mathrm{n}-1} \mathrm{f}\left(y_{1}\right)=\frac{n}{\beta}(1-$ $\mathrm{e}^{\left.-\mathrm{e}^{-\left(\frac{\mathrm{y}_{1}-\alpha}{\beta}\right)}\right)^{\mathrm{n}-1}} \mathrm{e}^{-\left(\frac{\mathrm{y}_{1}-\alpha}{\beta}\right)} \mathrm{e}^{-\mathrm{e}^{-\left(\frac{\mathrm{y}_{1}-\alpha}{\beta}\right)}}$

$-\infty<\alpha<\infty,-\infty<y_{1}<\infty, \beta>$.

To find $\mathrm{E}\left(\mathrm{Y}_{1}\right)$, we consider the $\mathrm{m}$. g. f. of $\mathrm{Y}$, $\mathrm{M}_{Y,}(\mathrm{t})=\mathrm{E} \quad\left(e^{t y_{1}}\right)=\int_{-\infty}^{\infty} e^{t y} \cdot \frac{n}{\beta}(1-$ $e^{\left.-e^{-\left(\frac{y_{1}-\alpha}{\beta}\right)}\right)^{n-1}} e^{-\left(\frac{y_{1}-\alpha}{\beta}\right)} e^{-e^{-\left(\frac{y_{1}-\alpha}{\beta}\right)}} d y_{1}$

Let $\mathrm{z}=\frac{y_{1}-\alpha}{\beta}$, then $\beta \mathrm{dz}=\mathrm{dy}$

$\mathrm{M}_{Y,}(\mathrm{t})=\mathrm{n} \int_{-\infty}^{\infty} e^{t(\alpha+\beta \mathrm{z})}(1-$

$\left.e^{-e^{-z}}\right)^{n-1} e^{-z} e^{-e^{-z}} d z$

$=\mathrm{n} e^{\alpha \mathrm{t}} \int_{-\infty}^{\infty}\left(e^{-z}\right)^{-\beta \mathrm{t}}\left(1-e^{-e^{-z}}\right)^{n-1}$

$e^{-z} e^{-e^{-z}} d z$

Let $\mathrm{w}=e^{-e^{-z}}$ and $\mathrm{d} \mathrm{w}=e^{-e^{-z}} e^{-z} d z$

$\mathrm{M}_{Y,}(\mathrm{t})=\mathrm{n} e^{\alpha \mathrm{t}} \int_{0}^{1}(-\ln (w))^{-\beta \mathrm{t}} \quad(1-$ $w)^{n-1} d w$

$$
=\mathrm{n}(-1)^{-\beta \mathrm{t}} e^{\alpha \mathrm{t}} \quad \int_{0}^{1}(\ln (w))^{-\beta \mathrm{t}}
$$

$(1+(-w))^{n-1} d w$

We know that

$$
\begin{aligned}
& (a+b)^{n}=\sum_{x=\cdot}^{n}\left(\begin{array}{l}
n \\
x
\end{array}\right) b^{x} a^{n-x}, \text { then } \\
& \mathrm{M}_{Y,}(\mathrm{t})=\mathrm{n}(-1)^{-\beta \mathrm{t}} e^{\alpha \mathrm{t}} \quad \int_{\cdot}^{1}(\ln (w))^{-\beta \mathrm{t}} \\
& \sum_{x=\cdot}^{n-1}\left(\begin{array}{c}
n-1 \\
x
\end{array}\right)(-w)^{x} d w \\
& \mathrm{M}_{Y,}(\mathrm{t})=\mathrm{n}(-1)^{-\beta \mathrm{t}} e^{\alpha \mathrm{t}} \sum_{x=\cdot}^{n-1}\left(\begin{array}{c}
n-1 \\
x
\end{array}\right)(-1)^{x} \\
& \int_{.}^{\prime}(\ln (w))^{-\beta \mathrm{t}} w^{x} d w
\end{aligned}
$$

$$
\begin{aligned}
& \quad=\mathrm{n}(-1)^{-\beta \mathrm{t}} e^{\alpha \mathrm{t}} \sum_{x=\cdot}^{n-1}\left(\begin{array}{c}
n-1 \\
x
\end{array}\right)(-1)^{x} \\
& \int_{0}^{1}(\ln (w))^{(1-\beta \mathrm{t})-1} w^{(x+1)-1} d w
\end{aligned}
$$

From Advanced Calculus, we have

$\int_{.}^{1}(\ln (w))^{k-1} w^{n-1} d w=\frac{\Gamma(k)}{n^{k}(-1)^{k+1}}$

$\mathrm{M}_{Y,}(\mathrm{t})=\mathrm{n}(-1)^{-\beta \mathrm{t}} e^{\alpha \mathrm{t}} \sum_{x=\cdot}^{n-1}\left(\begin{array}{c}n-1 \\ x\end{array}\right)(-1)^{x}$

$\frac{\Gamma(1-\beta \mathrm{t})}{(x+1)^{(1-\beta \mathrm{t})}(-1)^{(1-\beta \mathrm{t})+1}}$

$=\mathrm{n} \Gamma(1-\beta \mathrm{t}) e^{\alpha \mathrm{t}} \sum_{x=\cdot}^{n-1} \frac{\left(\begin{array}{c}n-1 \\ x\end{array}\right)(-1)^{x}}{(x+1)^{(1-\beta \mathrm{t})}}$

$\Phi(\mathrm{t})=\ln \left[\mathrm{M}_{Y},(\mathrm{t})\right]=\ln (\mathrm{n})+\ln [\Gamma(\mathrm{l}-\beta \mathrm{t})]+$ $\alpha t$

$+\ln \left[\sum_{x=\cdot}^{n-1}\left(\begin{array}{c}n-1 \\ x\end{array}\right)(-1)^{x}(x+1)^{(\beta t-1)}\right]$

$\Phi^{\prime}(\mathrm{t})=\alpha-\beta \Psi(1-\beta \mathrm{t})$

$+\frac{\sum_{x=.^{n}}^{n-1}\left(\begin{array}{c}n-1 \\ x\end{array}\right)(-1)^{x}(x+1)^{(\beta \mathrm{t}-1)} \beta \ln (x+1)}{\sum_{x=.}^{n-1}\left(\begin{array}{c}n-1 \\ x\end{array}\right)(-1)^{x}(x+1)^{(\beta \mathrm{t}-1)}}$

$\mathrm{E}(\mathrm{Y})=,\Phi(\cdot)=\alpha-\beta \Psi(1)$

$+\frac{\beta \sum_{x=\cdot}^{n-1}\left(\begin{array}{c}n-1 \\ x\end{array}\right)(-1)^{x}(x+1)^{(-1)} \ln (x+1)}{\sum_{x=\cdot}^{n-1}\left(\begin{array}{c}n-1 \\ x\end{array}\right)(-1)^{x}(x+1)^{(-1)}}$

$=\alpha-\beta \Psi$ (1)

$+\frac{\beta \sum_{x=\cdot}^{n-1}\left(\begin{array}{c}n-1 \\ x\end{array}\right) \frac{(-1)^{x}}{1+x} \ln (x+1)}{\sum_{x=\cdot}^{n-1}\left(\begin{array}{c}n-1 \\ x\end{array}\right) \frac{(-1)^{x}}{x+1}}$

But $\sum_{x=\cdot}^{n-1}\left(\begin{array}{c}n-1 \\ x\end{array}\right) \frac{(-1)^{x}}{x+1}=\frac{1}{n}$

$\mathrm{E}(\mathrm{Y})=,\alpha-\beta \Psi(1)$

$+\mathrm{n} \beta \sum_{x=\cdot}^{n-1}\left(\begin{array}{c}n-1 \\ x\end{array}\right) \frac{(-1)^{x}}{1+x} \ln (x+1)$

From Moments method, Smail and Myrtene $(r \ldots 0)[\wedge]$, is

$\mu=\mathrm{E}(\mathrm{X})=\alpha-\beta \Psi(1)=\alpha+\gamma \beta=\overline{\mathrm{X}}$

where $\Psi(\mathrm{z})=\frac{d}{d z} \ln (\Gamma(z))$ is known as digamma function and $\Psi(1)=-\gamma=-\cdot, \circ \vee V$ is called Euler's constant (Rand ( $\left.199 \mathrm{~V})\left[{ }^{\circ}\right]\right)$.

Now, we apply the modified moment method from eq.s ( $(\leqslant)$ and ( 10$)$ by setting:

$\hat{\mu}=\hat{\alpha}+\gamma \widehat{\beta}=\bar{X}$ and $E(Y)=$,$Y , at \alpha=\hat{\alpha}, \beta=$ $\widehat{\beta}$, we have to :

$\hat{\alpha}+\gamma \widehat{\beta}+\mathrm{n} \hat{\beta} \sum_{x=.}^{n-1}\left(\begin{array}{c}n-1 \\ x\end{array}\right) \frac{(-1)^{x}}{1+x} \ln (x+1)$ 


\section{Journal of Al-Nahrain University}

Science

$=\mathrm{Y}$,

$\hat{\alpha}+\gamma \widehat{\beta}=\bar{X}$

From eq.s ( 17 ) and ( $1 \mathrm{l})$, we obtain

$\widehat{\beta}=\frac{Y,-\bar{X}}{n \sum_{x=\cdot}^{n-1}\left(\begin{array}{c}n-1 \\ x\end{array}\right) \frac{(-1)^{x}}{x+1} \ln (x+1)}$

$\hat{\alpha}=\bar{X}-\gamma \widehat{\beta}$

Now, we will show that the estimators $\hat{\alpha}$ and $\widehat{\beta}$ are unbiased estimators for $\alpha$ and $\beta$ respectively, and its variances converge to zero as follows:

$\mathrm{E}(\hat{\beta})=\mathrm{E}\left[\frac{Y_{1}-\overline{\mathrm{X}}}{n \sum_{x=\cdot}^{n-1}\left(\begin{array}{c}n-1 \\ x\end{array}\right) \frac{(-1)^{x}}{x+1} \ln (x+1)}\right]$

$$
=\frac{\mathrm{E}\left(Y_{1}\right)-\mathrm{E}(\overline{\mathrm{X}})}{n \sum_{x=\cdot}^{n-1}\left(\begin{array}{c}
n-1 \\
x
\end{array}\right) \frac{(-1)^{x}}{x+1} \ln (x+1)}
$$

$\mathrm{E}(\widehat{\beta})=\frac{\mathrm{n} \beta \sum_{x=\cdot}^{n-1}\left(\begin{array}{c}n-1 \\ x\end{array}\right) \frac{(-1)^{x}}{1+x} \ln (x+1)}{n \sum_{x=\cdot}^{n-1}\left(\begin{array}{c}n-1 \\ x\end{array}\right) \frac{(-1)^{x}}{x+1} \ln (x+1)}$

$+\frac{\alpha+\gamma \beta-(\alpha+\gamma \beta)}{n \sum_{x=.}^{n-1}\left(\begin{array}{c}n-1 \\ x\end{array}\right) \frac{(-1)^{x}}{x+1} \ln (x+1)}=\beta$

$\operatorname{Bias}(\widehat{\beta})=E(\widehat{\beta})-\beta=\beta-\beta=$.

$\mathrm{E}\left(\hat{\beta}^{\Upsilon}\right)=\mathrm{E}\left[\left[\frac{Y,-\overline{\mathrm{X}}}{n \sum_{x=.}^{n-1}\left(\begin{array}{c}n-1 \\ x\end{array}\right) \frac{(-1)^{x}}{x+1} \ln (x+1)}\right]^{r}\right]$

$\mathrm{E}\left(\hat{\beta}^{r}\right)=\frac{\sigma^{r}+\left(\alpha+\gamma \beta+n \beta \sum_{x=\cdot}^{n-1}\left(\begin{array}{c}n-1 \\ x\end{array}\right) \frac{(-1)^{x}}{1+x} \ln (x+1)\right)^{r}-r E(Y, \bar{X})+\frac{\sigma^{r}}{n}+\mu^{r}}{\left[n \sum_{x=\cdot}^{n-1}\left(\begin{array}{c}n-1 \\ x\end{array}\right) \frac{(-1)^{x}}{x+1} \ln (x+1)\right]^{r}}$

$$
\begin{aligned}
\mathrm{E}(Y, \overline{\mathrm{X}}) & =\mathrm{E}\left(Y, \frac{1}{\mathrm{n}} \sum_{\mathrm{i}=1}^{\mathrm{n}}, \mathrm{X}_{\mathrm{i}}\right) & & \text { where } \mathrm{X}_{\mathrm{i}} \text { and } \mathrm{X}_{\mathrm{j}} \text { are independent } \\
& \left.=\frac{1}{\mathrm{n}} \mathrm{E}\left(\min \left(\mathrm{X}_{\mathrm{i}}\right) \sum_{\mathrm{i}=1}^{\mathrm{n}} \mathrm{X}_{\mathrm{i}}\right)\right) & & \mathrm{E}(Y, \overline{\mathrm{X}})=\frac{1}{\mathrm{n}}\left(\sigma^{r}+\mu^{r}+(\mathrm{n}-1) \mu^{r}\right) \\
& =\frac{1}{\mathrm{n}} \mathrm{E}\left(\left(\mathrm{X}_{\mathrm{i}}^{r}\right)+\sum_{\substack{j=1 \\
j \neq i}}^{n-1}\left(\mathrm{X}_{\mathrm{i}} \mathrm{X}_{\mathrm{j}}\right)\right) & & \text { Hence } \\
& =\frac{1}{\mathrm{n}}\left(\left(\operatorname{var}\left(\mathrm{X}_{\mathrm{i}}\right)+\left(\mathrm{E}\left(\mathrm{X}_{\mathrm{i}}\right)\right)^{r}\right.\right. & & \\
+\sum_{j=1}^{n-1} \mathrm{E} & \left.\mathrm{E}\left(\mathrm{X}_{\mathrm{i}}\right) \mathrm{E}\left(\mathrm{X}_{\mathrm{j}}\right)\right) & &
\end{aligned}
$$$$
=\frac{\mathrm{E}\left(Y,{ }^{r}\right)-r \mathrm{E}(Y, \overline{\mathrm{X}})+\mathrm{E}\left(\overline{\mathrm{X}}^{r}\right)}{\left[n \sum_{x=\cdot}^{n-1}\left(\begin{array}{c}
n-1 \\
x
\end{array}\right) \frac{(-1)^{x}}{x+1} \ln (x+1)\right]^{r}}
$$

$\mathrm{E}\left(\hat{\beta}^{r}\right)=\frac{\sigma^{r}+\left(\alpha+\gamma \beta+\mathrm{n} \beta \sum_{x=\cdot}^{n-1}\left(\begin{array}{c}n-1 \\ x\end{array}\right) \frac{(-1)^{x}}{1+x} \ln (x+1)\right)^{r}-r \frac{\sigma^{r}}{\mathrm{n}}-{ }^{r} \mu^{r}+\frac{\sigma^{r}}{n}+\mu^{r}}{\left[n \sum_{x=\cdot}^{n-1}\left(\begin{array}{c}n-1 \\ x\end{array}\right) \frac{(-1)^{x}}{x+1} \ln (x+1)\right]^{r}}$

$$
=\frac{\frac{(n-1)}{n^{r}} \sigma^{r}+\frac{r}{n}(\alpha+\gamma \beta)\left(\beta \sum_{x=\cdot}^{n-1}\left(\begin{array}{c}
n-1 \\
x
\end{array}\right) \frac{(-1)^{x}}{1+x} \ln (x+1)\right)+\left(\beta \sum_{x=\cdot}^{n-1}\left(\begin{array}{c}
n-1 \\
x
\end{array}\right) \frac{(-1)^{x}}{1+x} \ln (x+1)\right)^{r}}{\left[\sum_{x=\cdot}^{n-1}\left(\begin{array}{c}
n-1 \\
x
\end{array}\right) \frac{(-1)^{x}}{x+1} \ln (x+1)\right]^{r}}
$$

$\lim _{n \rightarrow \infty} E\left(\widehat{\beta}^{r}\right)=\beta^{r}$

From eq.s $(r \cdot)$ and $(r r)$, becomes

$\operatorname{Var}(\widehat{\beta})=\mathrm{E}\left(\widehat{\beta}^{r}\right)-[\mathrm{E}(\widehat{\beta})]^{r} \approx \beta^{r}-\beta^{r}=\cdot$

$E(\hat{\alpha})=E(\bar{X}-\gamma \widehat{\beta})=E(\bar{X})-\gamma E(\widehat{\beta})$

$$
=\alpha+\gamma \beta-\gamma \beta=\alpha
$$

$\operatorname{Bias}(\hat{\alpha})=E(\hat{\alpha})-\alpha=\alpha-\alpha=$.

From eq.s ( $r$ l) and ( $\left.r^{\circ}\right)$, we have

The estimators $\hat{\alpha}$ and $\hat{\beta}$ are unbiased estimators for $\alpha$ and $\beta$ respectively.

$\mathrm{E}\left(\hat{\alpha}^{r}\right)=\mathrm{E}\left((\overline{\mathrm{X}}-\gamma \widehat{\beta})^{r}\right)$

$=\mathrm{E}\left(\overline{\mathrm{X}}^{r}\right)-r \gamma \mathrm{E}(\overline{\mathrm{X}}) \mathrm{E}(\hat{\beta})+\gamma^{r}(\mathrm{E}(\widehat{\beta}))^{r}$ 
$\mathrm{E}\left(\hat{\alpha}^{r}\right)=\frac{\sigma^{r}}{n}+\mu^{r}-r \gamma \beta \mu+\gamma^{r} \beta^{r}$

$=\frac{\sigma^{r}}{n}+(\alpha+\gamma \beta)^{r}-r \gamma \beta(\alpha+\gamma \beta)+\gamma^{r} \beta^{r}$

$=\frac{\sigma^{r}}{n}+\alpha^{r}$

$\lim _{n \rightarrow \infty} E\left(\hat{\alpha}^{r}\right)=\alpha^{r}$

From eq.s ( $r \varepsilon)$ and $(r \tau)$, becomes

$\operatorname{Var}(\hat{\alpha})=\mathrm{E}\left(\hat{\alpha}^{r}\right)-[\mathrm{E}(\hat{\alpha})]^{r}$

$$
\approx \alpha^{r}-\alpha^{r}=\text {. }
$$

$\lim _{n \rightarrow \infty}$ m.s.e. $(\widehat{\beta})=\operatorname{Var}(\widehat{\beta})+(\operatorname{Bias}(\widehat{\beta}))^{r}=$.

$\lim _{n \rightarrow \infty}$ m.s.e. $(\hat{\alpha})=\operatorname{Var}(\hat{\alpha})+(\operatorname{Bias}(\hat{\alpha}))^{\Upsilon}=\cdot$

we conclusion that the estimators $\hat{\alpha}$ and $\widehat{\beta}$ are consistent.

\section{Confidence Interval Estimation for the Parameters of Gumbel Distribution}

In this section, we shall consider confidence interval estimation for the parameters $\alpha$ and $\beta$ based on the maximum likelihood method and modified moment method.

If the exact percentiles of the distribution of $\hat{\alpha}$ and $\widehat{\beta}$ were known, confidence intervals with confidence coefficients equal to the nominal confidence coefficients $1-\lambda$ could be found. The two-sides central $1 \cdots(1-\lambda) \%$ confidence intervals for $\alpha$ and $\beta$ based on maximum likelihood and modified moment estimators would be given by

$\left(\hat{\alpha}-z_{\left(1-\frac{\lambda}{\gamma}\right)}\left(\frac{\sigma}{n}\right)^{1 / r}, \hat{\alpha}+z_{\left(1-\frac{\lambda}{\gamma}\right)}\left(\frac{\sigma}{n}\right)^{1 / r}\right)$ and $(\widehat{\beta}-$ $\left.z_{\left(1-\frac{\lambda}{r}\right)}\left(\frac{\sigma}{n}\right)^{1 / r}, \widehat{\beta}+z_{\left(1-\frac{\lambda}{r}\right)}\left(\frac{\sigma}{n}\right)^{1 / r}\right)$ respectively, where $z_{\left(1-\frac{\lambda}{\gamma}\right)}$ represents to $1 \cdots(1-\lambda)$ of standard normal distribution Since the exact distribution of $\hat{\alpha}$ and $\hat{\beta}$ are unknown, an approximate confidence intervals may be found using the percentile approximation based on the maximum likelihood and modified moment estimators. (Hogg and Graig ( $9 \vee \wedge))[\varepsilon]$.

We take $90 \%$ confidence interval for $\hat{\alpha}$ and $\hat{\beta}$ based on maximum likelihood and modified moment estimators.

\section{Monte Carlo Results}

In the Monte Carlo simulation, $\mathrm{r}$ censored observations were taken with

$\mathrm{r}=\boldsymbol{O}(1) l \cdot(r) r \cdot$ from the sample $n=r$. and the simulation run size $\bullet \cdots$ was used.

Table ( 1 ) gives the values of bias, variance, mean square error, skewness, kurtosis and confidence intervals of the modified moment estimators.

Table ( $r$ ) gives the values of bias and variance of the maximum likelihood estimators. The variances are obtained from eq.s ( 9 ) and ( 11$)$.

Table ( $r)$ gives the values of mean square error and skewness of the maximum likelihood estimators.

Table $(\varepsilon)$. gives the values of kurtosis and confidence intervals of the maximum likelihood estimators. 
Table (')

Values of bias, variance, mean square error, skewness, kurtosis and confidence intervals for modified moment estimators.

\begin{tabular}{|c|c|c|c|}
\hline Moments & all r failure & Moments & all r failure \\
\hline $\operatorname{Bias}(\hat{\boldsymbol{\alpha}})$ & $-\cdot, \cdot 17 \cdot 9$ & $\operatorname{Bias}(\widehat{\beta})$ & $\cdot, .9919$ \\
\hline Var $(\hat{\boldsymbol{\alpha}})$ & •, .rqrV & $\operatorname{Var}(\widehat{\boldsymbol{\beta}})$ & -, MOYr \\
\hline M.S.E. ( $(\hat{\boldsymbol{\alpha}})$ & $\cdot, \cdot r V \cdot 0$ & M.S.E. $(\widehat{\boldsymbol{\beta}})$ & $\cdot, .5019$ \\
\hline Skew $(\hat{\alpha})$ & $-17,7 r v 1$. & Skew $(\widehat{\boldsymbol{\beta}})$ & $r \cdot 1, \Lambda 1 \cdot r \Lambda$ \\
\hline Kurt $(\hat{\boldsymbol{\alpha}})$ & $-Y \leqslant 10, Y q Y \cdot \theta$ & $\operatorname{Kurt}(\widehat{\beta})$ & $-11 V \Lambda, 0.7 Y$ \\
\hline $90 \%$ C. I. $(\hat{\boldsymbol{\alpha}})$ & $(-\cdot, \cdot \varepsilon \cdot 1 r,-\cdot, \cdot 1 r \cdot \tau)$ & $90 \%$ C. I. $(\widehat{\beta})$ & $\left(1, \cdot \wedge q \cdot 9,1,11 \sim v_{\cdot}\right)$ \\
\hline
\end{tabular}

Table (")

Values of bias and variance for maximum likelihood estimators.

\begin{tabular}{|c|c|c|c|c|}
\hline r failure & $\operatorname{Bias}(\widehat{\alpha})$ & $\operatorname{Bias}(\widehat{\boldsymbol{\beta}})$ & $\begin{array}{c}\text { Var }(\widehat{\alpha}) \text { practical } \\
\text { (theoretical) }\end{array}$ & $\begin{array}{c}\operatorname{Var}(\widehat{\beta}) \text { practical } \\
\text { (theoretical) }\end{array}$ \\
\hline 0 & $-\cdot, \wedge \vee q \vee$ &,.,$- 70 . \varepsilon$ & $\cdot, .91 \wedge 1(\cdot, \cdot 7 \leq \leqslant 9)$ & $\cdot, 1,710(\cdot, \cdot V R T \varepsilon)$ \\
\hline 7 & $-\cdot, \cdot \times 1 \cdot 7$ & $-\cdot, \cdot\{\wedge 9 q$ & 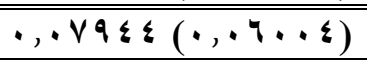 & 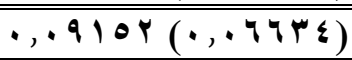 \\
\hline $\mathrm{V}$ & $-\cdot, \cdot 91 \mathrm{r}$ &,$- \cdot \varepsilon \cdot 9 V$ & $\cdot, \cdot T \operatorname{VOr}(\cdot, .0 T \vee 7)$ & $\cdot, \cdot \operatorname{VqIT}(\cdot, .0990)$ \\
\hline$\Lambda$ & $-\cdot, \cdot 4109$ & $-\cdots, \cdots \vee r \varepsilon$ & $\cdot,, 07 \leqslant Y(\cdot,, 0 \vee \leqslant \varepsilon)$ & $\cdot, .7104(\cdot, .014 \Lambda)$ \\
\hline 9 & $-\cdot, \cdot 1 \wedge r$. &,$+ \cdots 1 \cdot 9$ & $\cdot, .0 .9 \wedge(\cdot, .0 \vee \leqslant 1)$ & $\cdot, .07 \cdot \wedge(\cdot, .07 \leqslant V)$ \\
\hline 1. & $-\cdot, \cdot$ I YOr & $\cdot, .117 r$ & $\cdot, \cdot \Sigma \wedge \uparrow \cdot(\cdot, .0 \curlyvee \wedge \vee)$ & $\cdot, .0 \leqslant 9 \cdot(\cdot, .044 \wedge)$ \\
\hline Ir & $-\cdot, \cdots 7 r$ & $\cdot, .1147$ &., $.4909(., .0741)$ & 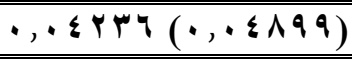 \\
\hline $1 \varepsilon$ & $-\cdots, \ldots 0 \leq$ & $\cdot, \cdot r I V 0$ & $\cdot, \cdot r \Delta V \cdot(\cdot, \cdot O V / 7)$ & 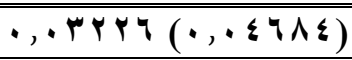 \\
\hline 17 & $\cdot, \cdots r \leqslant V$ & 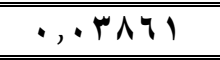 & 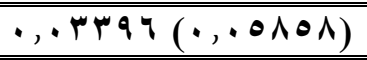 & 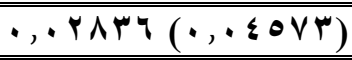 \\
\hline 11 &.,$\ldots 00 \mathrm{~V}$ & $\cdot, \cdot \varepsilon Y 00$ & $\cdot, \cdot r r \varepsilon \cdot(\cdot, \cdot 7.79)$ & $\cdot, \cdot, Y \leq r \checkmark(\cdot, \cdot, \leqslant 0 \leqslant V)$ \\
\hline$r \cdot$ & $9, \ldots 911$ & $\cdot, \cdot \leq \leqslant q V$ & $\cdot, \cdot r \mid \vee v(\cdot, \cdot 7 r q 0)$ & 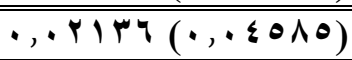 \\
\hline
\end{tabular}

Table (")

Values of mean square error and skewness for maximum likelihood estimators.

\begin{tabular}{|c|c|c|c|c|}
\hline r failure & M.S.E. $(\widehat{\alpha})$ & M.S.E. $(\widehat{\boldsymbol{\beta}})$ & Skew $(\widehat{\alpha})$ & Skew $(\widehat{\boldsymbol{\beta}})$ \\
\hline 0 & 0,99900 & $\cdot, 11 \cdot r \wedge$ & $-9,91.74$ & $r \varepsilon, v \circ r \Delta q$ \\
\hline 7 & $\cdot, \cdot \wedge \leq \leq 9$ &., .9491 & $-1 \cdot, q q \leq 1 r$ & 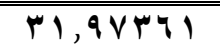 \\
\hline $\mathrm{V}$ & $\cdot, \cdot V I Y \Lambda$ & $\cdot, \cdot \vee \vee \wedge \wedge$. & $-\mid r, \cdot \varepsilon r \cdot 1$ & $\varepsilon r, 79.00$ \\
\hline$\Lambda$ & $\cdot, \cdot O V \leqslant Y$ &., .9171 & $-14,197 . \leq$ & $T \varepsilon, T Y O Y \leq$ \\
\hline 9 &., .0111 &., $091 \leq$ & $-1 \varepsilon, r \Delta q r V$ & $V V, 0 \leq r \ldots$ \\
\hline 1. & $\cdot, \cdot \leq \wedge \mu q$ &., $.00 . r$ & $-10, \leq \wedge \leq 0 \wedge$ & A., $\leqslant \Lambda .01$ \\
\hline $1 \%$ & +,.,rq9r & 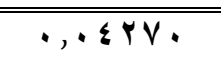 & $-19,19 \cdot 19$ & $|r|, \cdot r \leqslant q r$ \\
\hline $1 \varepsilon$ & $\cdot, \cdot r \circ V$. & -, MTYV & $-1 \varepsilon, 0 \wedge \Delta \mu \wedge$ & $1 \wedge q, \leq V 1 Y q$ \\
\hline 17 & $\cdot, . \mathrm{rMqV}$ & $\cdot, \cdot r 9 \wedge 0$ & $-1 r, \wedge \neg r v \leq$ & YrE, \\
\hline 11 & - , $\cdot r r \leq r$ & -, YYIV & $-|r, Y| \otimes \wedge \theta$ & PqV, Yrr.0 \\
\hline
\end{tabular}




\begin{tabular}{|c||c||c||c||c|}
\hline$r \cdot r, r I V q$ & $\cdot, r r r \wedge$ & $-11,0 r V V r$ & $r q \varepsilon, q 17 r V$ \\
\hline
\end{tabular}

Table ( $\varepsilon)$

Values of kurtosis and confidence intervals for maximum likelihood estimators.

\begin{tabular}{|c|c|c|c|c|}
\hline r failure & Kurt $(\widehat{\alpha})$ & Kurt $(\widehat{\boldsymbol{\beta}})$ & $90 \%$ C.I. ( $\widehat{\alpha})$ & 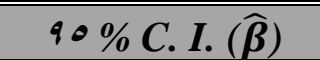 \\
\hline 0 & - ror, rrqqV & $-77,91 r \leq \varepsilon$ & $\begin{array}{c}(-\cdot, 11 \cdot r 7,-\cdot, \cdot 707 \\
1)\end{array}$ & $\begin{array}{c}(\cdot, 91.99, \cdot, 90 \wedge 9 \\
9)\end{array}$ \\
\hline 7 & $-r \varepsilon r, \cdot \Lambda v q r$ & $-q V, r V \leq 1 \wedge$ & $\begin{array}{c}(-\cdot, \cdot 91 \vee q,-\cdot, \cdot 0 \cdot r \\
r)\end{array}$ & $\begin{array}{c}(\cdot, q \vee \wedge \vee q, \cdot, q \vee r r \\
\cdot)\end{array}$ \\
\hline v & $-\varepsilon \neg 7, r \leq \Lambda r$ & $-|\varepsilon \theta, \mu| \wedge \mu \Delta$ & $\begin{array}{c}c(-\cdot, \cdot \Lambda \cdot \varepsilon V,-\cdot, \cdot \varepsilon r r \\
\varepsilon)\end{array}$ & $\begin{array}{c}(\cdot, q r \wedge \vee r, \cdot, q \vee q r \\
r)\end{array}$ \\
\hline$\Lambda$ & $-V M \cdot, r r I V V$ & $-r \diamond \bullet, \neg \cdot r q \wedge$ & $\begin{array}{c}c(-\cdot, \cdot \leq q \cdot v,-\cdot, \cdot 1 \leq 1 \\
v)\end{array}$ & $\begin{array}{c}(\cdot, 9 \vee \leq 0 ., 1, \cdot 11 . \\
1)\end{array}$ \\
\hline 9 & $-q r r, q 1 \cdot v r$ & $-r p v, \Lambda \cdot . q q$ & $\begin{array}{c}(-\cdot,+r \leq \Lambda),-\cdot,+\cdots 10 \\
q)\end{array}$ & $\begin{array}{c}(\cdot, 99 \cdot 9 v, 1, \cdot r 00 \\
1)\end{array}$ \\
\hline 1. & $\begin{array}{c}-1 \cdot \varepsilon q, \mu 0 \ldots \\
\Lambda\end{array}$ & $-r \leq 0, q \leq r \leq r$ & $\begin{array}{c}c(-\cdot, r \wedge q v,-\cdot, \cdot r q \\
r)\end{array}$ & $\begin{array}{c}(\cdot, q q \leq r q, 1, \cdot r \wedge \wedge \\
q)\end{array}$ \\
\hline Ir & $\begin{array}{c}-10 \varepsilon \cdot, r . q 9 \\
r\end{array}$ & $-\Delta q \vee, r q r \wedge l$ & $(-\cdot, \cdot r \cdot q \varepsilon, \cdot, \cdot \wedge r \varepsilon)$ & $\begin{array}{c}(1, \cdots r \circ r, 1, \cdot r \mu \Lambda \\
\cdot)\end{array}$ \\
\hline $1 \varepsilon$ & $19 \leq 0,7 \wedge \leq \Delta \vee$ & $\frac{-}{1 \cdot \wedge q, q 1 \% \cdot q}$ & $(-\cdot, \cdot 1 \varepsilon \varepsilon \varepsilon, \cdot, \cdot \mid r \mu v)$ & $\begin{array}{c}(1,1 \wedge 0 \leq, 1, \cdot \leq \leq 9 \\
v)\end{array}$ \\
\hline 17 & $\begin{array}{c}- \\
r \backslash \wedge r, \mid q \vee \backslash V \\
\end{array}$ & $1 \leq \varepsilon \leq, 79197$ & $(-\cdot, \cdot 1 \cdot \cdot q, \cdot, \cdot 1 \vee \cdot r)$ & $\begin{array}{c}(1, \cdot r q r \mu, 1,01 . \\
\cdot)\end{array}$ \\
\hline 11 & $\begin{array}{c} \\
\text { rrqu, q09q1 } \\
\end{array}$ & $19 \wedge v, 9 \cdot 10 \%$ & $(-\cdot, \cdot \vee \vee \wedge \vee, \cdot, \cdot 19 \cdot r)$ & $\begin{array}{c}(1, \cdot, \cdot v, 1, \cdot 0 \varepsilon . \\
\varepsilon)\end{array}$ \\
\hline$r$ & - & $\frac{-}{r}, \Lambda, \vee r \cdot 0$. & $(-\cdot, \cdot 999, \cdot, \cdot 19 r r)$ & $\begin{array}{c}(1, \cdot r \varepsilon r 1,1, .00 V \\
r)\end{array}$ \\
\hline
\end{tabular}

\section{Conclusion}

In this paper, we use maximum likelihood method and modified moment method to estimate the parameters of Gumbel (maximum extreme value) distribution of right censored data of type II. The bias, variance and mean square error are decreasing rapidly as the sample size of censored data increase in maximum likelihood method. The values of the practical variances of maximum likelihood estimators are very close to the theoretical values given by eq.s ( 9 ) and (1), and the difference approach to zero, as the confidence limits of interval estimation become close to the true value of the distribution parameters as sample size increase. The skewness is increasing in small sample to middle the sample, and then decreasing in large sample, whereas the kurtosis is increasing faster as sample size increase.

While in modified moment method, the estimators and other moments in all sample size of censored data increase are same it because the sample is no change in order the sample of censored data increase according to order statistic theory.

Adding to, we show that the estimators $\hat{\alpha}$ and $\widehat{\beta}$ are unbiased estimators for $\alpha$ and $\beta$ respectively given by eq.s $\left(r_{\bullet}\right)$ and $(T \varepsilon)$, and its variances converge to zero given by eq.s $(\Upsilon r)$ and $(Y V)$, which the estimators $\hat{\alpha}$ and $\hat{\beta}$ are consistent. The estimators and other moments in maximum likelihood method are very close from modified moment method, but maximum likelihood method is the best than modified moment method. All this indicate that the 


\section{Journal of Al-Nahrain University}

Science

estimators distribution approach rapidly to normal in two methods.

\section{Reference}

['] Balakrishnan N., Kannan N., Lin C.T., and Wu S.J., "Inference for the extreme value distribution under progressively Type-II censoring", Journal of Statistical Computation \& Simulation, Vol. V $\leqslant$, ro $\leqslant 0, r \ldots \varepsilon$.

[r] El Sherpieny E. A., Assar S. M. and Amer N. M., "Parameters Estimation of Generalized Extreme Value Distribution under Progressive Type II Censored", Asian Journal of Applied Sciences, Vol. ' $(r),{ }^{\top}{ }_{-}$ $V T, Y \cdot I r$.

[r] Fard M. N. and Holmquist B., "Powerful goodness-of-fit tests for the extreme value distribution", Chilean Journal of Statistics, Vol. ₹(1), $00-T V, r \cdot 1 r$.

[₹] Hogg R. and Graig A., "Introduction to Mathematical Statistic", Forth Edition, Macmillan Publishing O., New York, Inc., 19VA.

[०] Rand Q.Z., "Approximation to the incomplete gamma integral with random variates procedures Simula“, M. Sc. Thesis, AL-Nahrain University, Baghdad, Iraq, 199V.

[`] Salinas V., Pérez P., González E. and Vaquera H., "Goodness of Fit Tests for the Gumbel Distribution with Type II right Censored data", Revista Colombiana de Estadística, Vol. ro(r), $\_\cdot q_{-} \leqslant r \leqslant, r \cdot \mid r$.

$[\vee$ ] Sheng Z., "Order-statistics-based Inferences for Censored Lifetime Data and Financial Risk Analysis", A thesis submitted for the degree of Doctor of Philosophy, Brunel University, London, r.Ir.

[^] Smail M. and Myrtene C., "Estimation parameters of Gumbel distribution using the methods of moments, probability weighted moments and maximum likelihood", Revista De Matematica: Teoria Y Aplicaciones, Vol. I T, 101_107, Y..0.

[9] Thompson E. M., Hewlett J. B., Baise L. G. and Vogel R. M., "The Gumbel hypothesis test for left censored observations using regional earthquake records as an example“, National Hazards
Earth System Sciences, Vol. 11, $110_{-}$I Y $r \cdot 11$.

[1.] Wu J. W., Chen S. C., Lee W. C. and Lai H. Y., "Weighted Moments Estimators of the Parameters for the Extreme Value Distribution Based on the Multiply Type II Censored Sample“, The Scientific World Journal, Vol. $r \cdot 1 r, 1-\Lambda, r \cdot 1 r$.

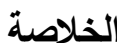

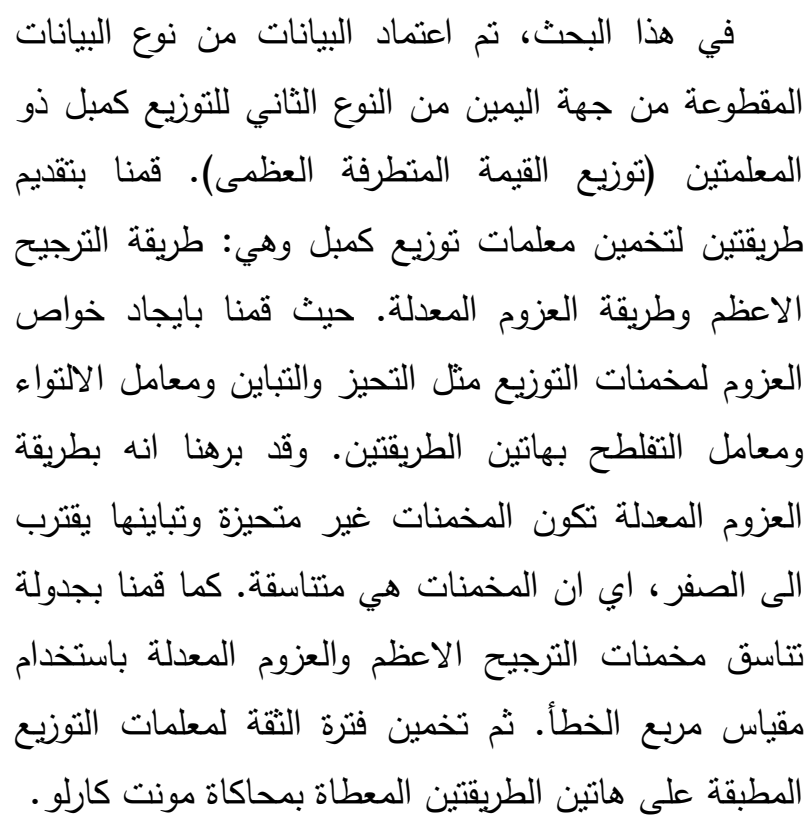


Fadi A. Shaayo

190 